\title{
Search for anomalously coupling and fermiophobic Higgs Bosons
}

\author{
Christophe Delaere ${ }^{* ;}$ on behalf of the LEP collaborations \\ Universite catholique de Louvain, Belgium \\ E-mail: christophe.delaere@cern.ch
}

Searches for fermiophobic Higgs boson decaying into photon or weak boson pairs have been performed at LEP with the data collected at center of mass energies up to $209 \mathrm{GeV}$. A summary of several analyses allowing to cover some hundred different final state topologies is presented. No statistically significant evidence of fermiophobic Higgs boson has been found. A lower limit on the Higgs boson mass is presented. More generally, anomalous couplings of the Higgs boson are searched for through the processes $\mathrm{e}^{+} \mathrm{e}^{-} \rightarrow \mathrm{H}^{0} \gamma, \mathrm{e}^{+} \mathrm{e}^{-} \rightarrow \mathrm{e}^{+} \mathrm{e}^{-} \mathrm{H}^{0}$ and $\mathrm{e}^{+} \mathrm{e}^{-} \rightarrow \mathrm{H}^{0} \mathrm{Z}$ by the $\mathrm{L} 3$ collaboration. The Higgs decay channels $\mathrm{H}^{0} \rightarrow \mathrm{ff}, \mathrm{H}^{0} \rightarrow \gamma \gamma, \mathrm{H}^{0} \rightarrow \mathrm{Z} \gamma$ and $\mathrm{H}^{0} \rightarrow \mathrm{WW}^{(*)}$ are considered and no evidence is found for anomalous Higgs production or decay and limits are put.

International Europhysics Conference on High Energy Physics

July 21st - 27th 2005

Lisboa, Portugal

* Speaker.

${ }^{\dagger}$ FNRS research fellow 


\section{Introduction}

A Higgs model incorporating two doublets of complex scalar fields generates five scalar Higgs bosons, three of which are neutral. For type-I models, and for certain choices of the model parameters, one of these neutral scalars provides mass only to the fermions and the other couples exclusively to the bosons, i.e. is a "fermiophobic" Higgs boson.

At the Large Electron-Positron collider, the search for a fermiophobic Higgs boson has been primarily carried out in the $\mathrm{H} \rightarrow \gamma \gamma$ channel, in which the Higgs boson couples to photons via a W loop [1]. For fermiophobic Higgs bosons heavier than $90 \mathrm{GeV}$, the predicted $\mathrm{H} \rightarrow \gamma \gamma$ branching ratio becomes small relative to the predicted $\mathrm{H} \rightarrow \mathrm{WW}$ branching ratio motivating a search in this new channel $[2,3]$.

Anomalous $\mathrm{H}^{0} \gamma \gamma$ and $\mathrm{H}^{0} \mathrm{Z} \gamma$ couplings are also searched for by the L3 collaboration [4], with sensitivity to Higgs masses up to the center-of-mass energy of the collision $\left(m_{\mathrm{H}}<\sqrt{s}\right)$.

\section{Searches for Higgs boson decaying into photons}

All of the LEP experiments search for hadronic, leptonic, and missing energy (neutrino) decay modes of the associated $\mathrm{Z}$ boson in the production channel $e^{+} e^{-} \rightarrow H Z$ [1].

The selected events are used to set an upper limit on the di-photon branching ratio of particles produced in association with a $Z$. Figure 1 shows the $95 \% \mathrm{CL}$ upper limit on $B\left(\mathrm{~h}^{0} \rightarrow \gamma \gamma\right) \times$ $\sigma\left(\mathrm{e}^{+} \mathrm{e}^{-} \rightarrow \mathrm{h}^{0} \mathrm{Z}^{0}\right) / \sigma(\mathrm{SM})$ obtained by combining the candidate events from the four experiments. The mass at which $B\left(\mathrm{~h}^{0} \rightarrow \gamma \gamma\right)=1$ is excluded at the $95 \%$ C.L. is $117.2 \mathrm{GeV}$.

Also shown in the Figure is the $\mathrm{h}^{0} \rightarrow \gamma \gamma$ branching ratio in the Standard Model computed with the fermionic couplings switched off. The benchmark fermiophobic lower mass limit is obtained where the predicted branching ratio crosses the upper-limit curve. For the combined data from the four experiments, the 95\% CL lower mass limit for a benchmark fermiophobic Higgs boson is set at $109.7 \mathrm{GeV}$.

\section{Searches for Higgs boson decaying into $\mathrm{W}$ bosons}

Processes involving a Higgs boson decaying into $\mathrm{W}$ bosons at LEP are characterized by two $\mathrm{W}$ bosons and two fermions, originating from a $\mathrm{Z}$ boson in the dominating Higgs-strahlung diagram. In the Higgs boson mass range kinematically accessible at LEP, one of the virtual W's is expected to be near on-shell, and the other to have a much smaller mass and energy.

The full spectrum of $\mathrm{HZ} \rightarrow$ WWZ contains a total of 96 topologies depending on the decays of the W's and the Z. These final states were pooled in 13 exclusive selections by the ALEPH collaboration, depending on the number of jets, hard leptons and soft leptons in the final state [2]. The L3 collaboration concentrated on the 8 main analyses, covering 93\% or the branching ratio [3].

The best limit is obtained by combining the present results with the one previously published exploiting $\mathrm{H} \rightarrow \gamma \gamma$. The $95 \%$ C.L. limit on $\mathrm{BR}_{\text {bosons }}$ ( that represents the total Higgs branching fraction to pairs of gauge bosons) is determined at each point of the $m_{H}$ versus $\mathrm{R}_{\gamma \gamma}$ plane, resulting in the exclusion curves of Figure 2. For that purpose, $\mathrm{R}_{\gamma \gamma}$ is defined as the fraction of fermiophobic decays into photon pairs. In the benchmark fermiophobic scenario, the fermiophobic Higgs boson 


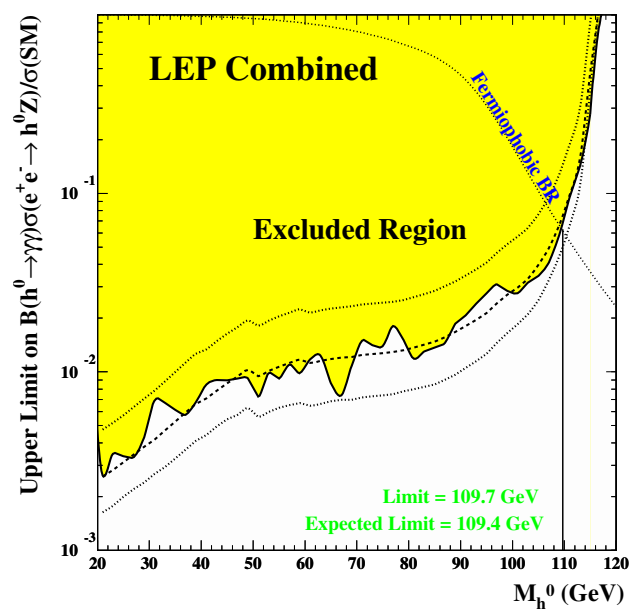

Figure 1: Combined LEP experimental limits for Higgs bosons decaying into di-photons. The 95\% confidence level upper limit on $B\left(\mathrm{~h}^{0} \rightarrow \gamma \gamma\right) \times \sigma\left(\mathrm{e}^{+} \mathrm{e}^{-} \rightarrow \mathrm{h}^{0} \mathrm{Z}^{0}\right) / \sigma(\mathrm{SM})$ is shown as a function of Higgs mass. Also shown (dotted line) is the branching fraction obtained for the benchmark fermiophobic model.

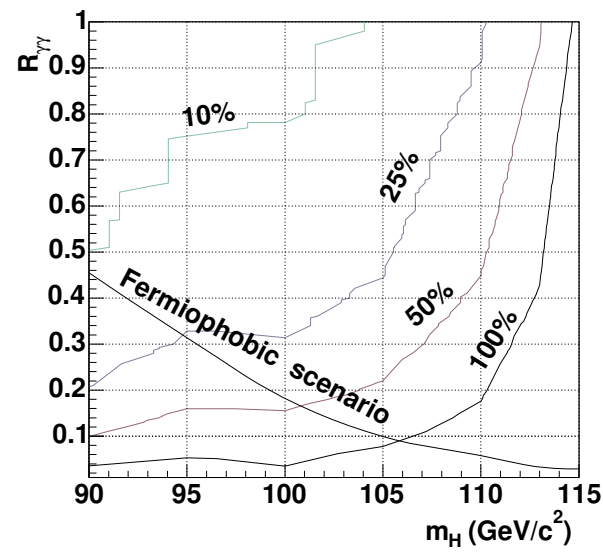

Figure 2: The $95 \%$ C.L. limit for $\mathrm{BR}_{\text {bosons }}$ as a function of $m_{H}$ and $\mathrm{R}_{\gamma \gamma}$, obtained by the ALEPH collaboration. The solid lines indicate the upper limit of exclusion regions. From [2].

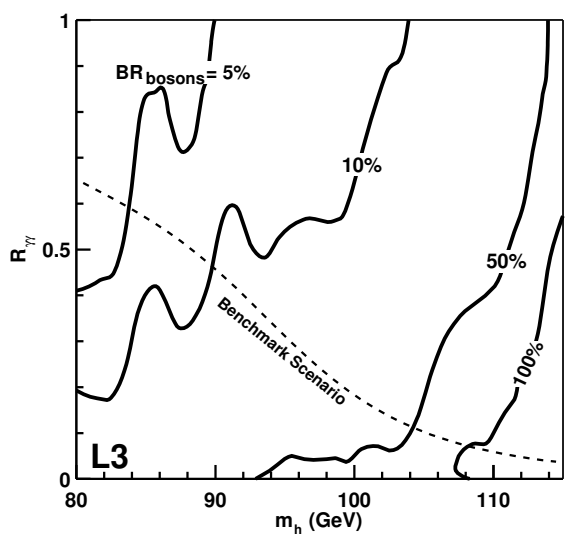

Figure 3: The $95 \% \mathrm{CL}$ limit for $\mathrm{BR}_{\text {bosons }}$ as a function of $m_{H}$ and $\mathrm{R}_{\gamma \gamma}$, obtained by the L3 collaboration. The solid lines indicate the upper limit of exclusion regions. From [3]

is excluded up to $105.8 \mathrm{GeV} / \mathrm{c}^{2}$ by the ALEPH collaboration, and up to $108.3 \mathrm{GeV} / \mathrm{c}^{2}$ by the $\mathrm{L} 3$ collaboration. In Figures 2 and 3, this corresponds to the crossing point between the " $B R_{\text {bosons }}=$ $100 \% "$ line and the "Fermiophobic scenario" line.

\section{Search for anomalous couplings in the Higgs sector}

The Standard Model can be extended, via a linear representation of the $S U(2)_{L} \times U(1)_{Y}$ symmetry breaking mechanism, to higher orders where new interactions between the Higgs boson and gauge bosons become possible. These modify the production mechanisms and decay properties of the Higgs boson. The couplings in a CP-invariant Lagrangian are conveniently parametrized 
introducing five dimensionless parameters $d, d_{B}, \Delta g_{1}^{Z}, \Delta \kappa_{\gamma}$ and $\delta_{Z}$ The couplings $\Delta g_{1}^{Z}$ and $\Delta \kappa_{\gamma}$ are commonly used in the context of $\mathrm{e}^{+} \mathrm{e}^{-} \rightarrow$ WW studies. Limits on the parameter $\xi=\left(1+\delta_{\mathrm{Z}}\right)$, which quantifies deviations in the magnitude of the $\mathrm{H}^{0} \mathrm{ZZ}$ and $\mathrm{H}^{0} \mathrm{WW}$ couplings have already been discussed in sections 3 .

The analyses performed by the L3 collaboration over the different Higgs production mechanisms and decay channels show that the experimental data agree with the Standard Model predictions [4]. Exclusion limits for each individual coupling are derived as a function of the Higgs mass. Assuming the absence of large anomalous WWZ and WW $\gamma$ couplings, the maximal partial widths and branching fractions of the decays $\mathrm{H}^{0} \rightarrow \mathrm{Z} \gamma$ and $\mathrm{H}^{0} \rightarrow \gamma \gamma$ are computed (Figure 4). The results are consistent with the tree level Standard Model expectations $\Gamma\left(\mathrm{H}^{0} \rightarrow \mathrm{Z} \gamma\right) \approx \Gamma\left(\mathrm{H}^{0} \rightarrow \gamma \gamma\right) \approx 0$.
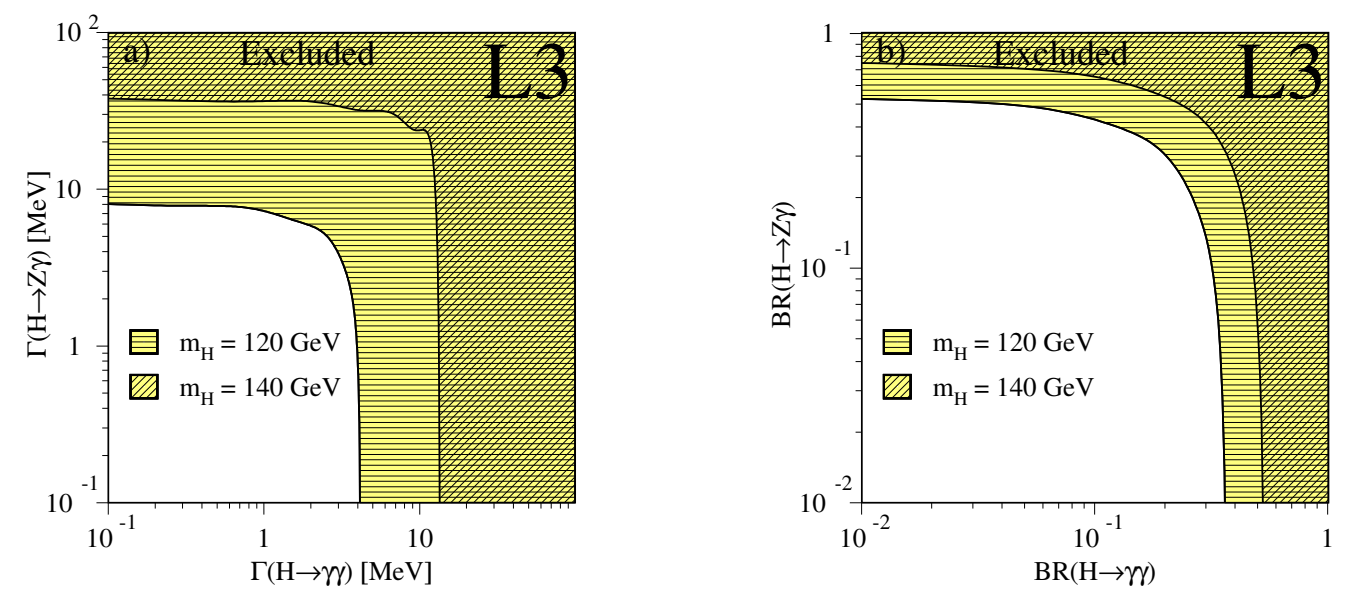

Figure 4: Regions excluded at 95\% CL for: a) the partial widths $\Gamma\left(\mathrm{H}^{0} \rightarrow \mathrm{Z} \gamma\right) v s . \Gamma\left(\mathrm{H}^{0} \rightarrow \gamma \gamma\right)$ and b) the branching fractions $\operatorname{Br}\left(\mathrm{H}^{0} \rightarrow \mathrm{Z} \gamma\right) v s$. $\operatorname{Br}\left(\mathrm{H}^{0} \rightarrow \gamma \gamma\right)$ in presence of the $d$ and $d_{B}$ anomalous couplings. Two values of the Higgs boson mass are considered. From [4].

\section{Acknowledgments}

It is a pleasure to congratulate my colleagues from the accelerator divisions for the outstanding operation of LEP 2. We are indebted to the engineers and technicians for their contributions to the excellent performance of each detector. The author would like to thank the Belgian FNRS for supporting him as research fellow.

\section{References}

[1] LEP Collaborations, "Searches for Higgs Bosons Decaying into Photons: Combined Results from the LEP Experiments", LHWG Note/2002-02, 12 July '02

[2] ALEPH Collaboration, Search for WW decay of a Higgs boson produced in association with a fermion pair in e+e- collisions at LEP. Aleph 2003-018. (2003); to be published.

[3] L3 collaboration, Search for a Higgs Boson Decaying to Weak Boson Pairs at LEP, Phys. Lett. B568 (2003) 191-204

[4] L3 collaboration, Search for Anomalous Couplings in the Higgs Sector at LEP, CERN-PH-EP/2004-002, Phys. Lett. B589 (2004) 89-102. 